\title{
АНТИТРОМБОТИЧЕСКАЯ ТЕРАПИЯ
}

\section{РОЛЬ АНТИТРОМБИНА В ДИАГНОСТИКЕ \\ И ИНТЕНСИВНОЙ ТЕРАПИИ ОСТРОГО ДВС-СИНДРОМА}

\section{(обзор литературы и данные реальной клинической практики)}

\author{
Н.А. ВОРОБЬЕВА, д.м.Н., профессор, \\ Северный филиал Национального медицинского исследовательского \\ центра гематологии Минздрава России
}

\begin{abstract}
В статье рассматриваются вопросы патогенеза, диагностики и интенсивной терапии острого ДВС-синдрома на фоне дисфункции антикоагулянтной системы. Показана роль ранней лабораторной диагностики снижения активности антитромбина III для оптимизации интенсивной терапии острого ДВС-синдрома. С позиции доказательной медицины и реальной клинической практики продемонстрирована возможность использования концентрата антитромбина III. КлЮчЕВЫЕ СЛОВА: ДВС-синдром, антитромбин, полиорганная недостаточность, диагностика, интенсивная терапия
\end{abstract}

\section{ROLE OF ANTITHROMBIN IN DIAGNOSTICS AND INTENSIVE THERAPY OF ACUTE DIC-SYNDROME (review of literature and evidence of actual clinical practice)}

N.A. VOROBIEVA, MD, Prof.

North Branch of the Federal State Budgetary Institution

of the National Medical Research Center of Hematology of the Ministry of Health of Russia

The article considers the issues of pathogenesis, diagnostics and intensive therapy of acute DIC-syndrome with underlying dysfunction of anticoagulant system. It also shows the role of early laboratory diagnostics of decreased antithrombin III activity for optimization of intensive therapy of acute DIC syndrome. The possibility of using antithrombin III concentrate has been demonstrated from the perspective of evidence-based medicine and real clinical practice.

KEYWORDS: DIC-syndrome, antithrombin, multi-organ failure, diagnosis, intensive care

дним из самых тяжелых и серьезных дисбалансов системы гемостаза в практике критической медицины является острый ДВС-синдром. Искусство врача-интенсивиста, реаниматолога, хирурга, акушера часто определяется именно успехами в интенсивной терапии данного состояния.

Как известно, ДВС-синдром является неспецифическим универсальным клинико-патологическим процессом, осложняющим течение самых многочисленных заболеваний, а также причиной высокой летальности, что определяет междисциплинарность и актуальность данной проблемы для врачей всех специальностей. Многочисленные фундаментальные работы отечественных и зарубежных авторов посвящены вопросам этиологии, патогенеза, диагностики и интенсивной терапии острого ДВС-синдрома. Немаловажно отметить, что ДВС-синдром осложняет и предопределяет 
прогноз ряда форм сепсиса, акушерской патологии, инфекционно-септических состояний, травм, ожогов, оперативных вмешательств и всех критических состояний, сопровождающихся синдромом полиорганной недостаточности (ПОН) [1, 2].

Являясь одной из основных составляющих ПОН, острый ДВС-синдром в значительной степени предопределяет исход заболевания, а лечение его представляет трудную задачу и далеко не всегда бывает успешным. При этом необходимо отметить, что исход фоновых заболеваний в значительной мере зависит от степени ургентности и объема интенсивной терапии ДВС-синдрома. Однако зачастую недостаточные представления о патогенезе и клинических проявлениях ДВС-синдрома приводят к неполноценной и несвоевременной диагностике данного осложнения и фрагментарности последующих мероприятий, направленных на коррекцию нарушений системы гемостаза.

Как известно, кроме активации коагуляции в патогенезе ДВС-синдрома значимую роль играет дисфункция антикоагулянтной системы. В первую очередь нарушаются механизмы регуляции образования тромбина на фоне дефицита AT III в силу ряда причин. Во-первых, активность АТ III снижается вследствие его продолжающегося потребления на фоне образования тромбина и других активированных протеаз, с которыми AT III способен образовывать комплексы [3]. Во-вторых, АT III разрушается эластазой активированных нейтрофилов. И наконец, вследствие развития дисфункции печени нарушается синтез AT III и происходит экстравазальная утечка данного ингибитора протеаз.

По мнению большинства авторов, наиболее важным тестом для диагностики и оценки тяжести ДВС-синдрома является мониторинг активности AT III [1, 2, 4-8], так как при этом патологическом состоянии накапливается значительное количество активированных форм протеолитических ферментов, на нейтрализацию которых и используется данный антикоагулянт. Исследование активности АТ III в динамике ДВС-синдрома позволяет судить о его потреблении активированными факторами свертывания крови и об эффективности терапии, в частности гепарином, СЗП, концентратами АТ III и антипротеазами, т. к. лечебный эффект гепарина резко ослабляется при уменьшении активности AT III $[1,9]$. Немаловажно отметить, что в 2016 г. японские авторы включили обязательную лабораторную оценку функциональной активности AT III в обновленную шкалу диагностики ДВС-синдрома [8].

Из литературных данных известно, что существуют как наследственные, так и приобретенные причины дефицита антитромбина III. Впервые наследственный дефицит антитромбина, клинически проявляющийся тромбофилией, был описан Egeberg в 1965 г. По некоторым данным, присутствие наследственного дефицита AT III в общей популяции составляет до 0,003\% [9], а среди пациентов с клинически значимыми эпизодами венозного тромбоэмболизма до 3-5\% [10, 11]. В настоящее время хорошо изучено два основных типа наследственного дефицита AT III. Так, первый тип обусловлен низким уровнем АТ III с нормальной функциональной активностью, а при втором типе AT III присутствует в плазме крови в нормальном количестве, но его функциональная активность нарушена. В большинстве случаев это обусловлено нарушением процесса связывания AT III с гепарином, хотя описаны и другие изменения структуры молекулы АТ Ш.

Генетически обусловленный дефицит АТ III связан с наследованием дефектного гена по аутосомному доминантному механизму, что сопровождается повышенным риском как венозного, так и артериального тромбоза с манифестацией клинических проявлений в молодом возрасте. Гомозиготное носительство наследст- 
венного дефицита AT III представляет собой весьма редкую патологию, сопровождающуюся манифестацией тромбообразования у новорожденных или в раннем детстве. Гетерозиготный дефицит AT III приводит к развитию венозного тромбоза, который клинически проявляется в возрасте после двадцати лет. Дефектный ген наследуется по аутосомному доминантному типу, при этом у кровных родственников обнаруживается различная генетическая патология. Дефект чаще возникает в процессе трансляции или после процессинга в результате замещения одной аминокислоты в начале $\beta$-цепи пептида. Замещение, которое приводит к присутствию полярных аминокислот в данной локализации («ген Wibble»), обуславливает снижение функциональной активности антитромбина III. Другие замещения связаны с менее выраженным снижением активности энзима. Клинически «ген Wibble» ассоциируется с повышенным риском тромбоза, который проявляется в более раннем возрасте, как правило, от десяти до двадцати лет [12].

В практике интенсивной терапии и медицины критических состояний наибольшее распространение, а значит, и клиническое значение имеет приобретенный дефицит АТ III, который возникает вследствие его повышенного потребления. Основная причина данного состояния заключается в чрезмерной активации системы коагуляции крови при ДВС-синдроме, сепсисе, ССВО. Любые состояния, сопровождающиеся гиперкоагуляцией, повреждением эндотелия, присутствием антифосфолипидных антител, тромбинемии, приводят к повышенному потреблению АТ III. Также известны другие известные механизмы приобретенного дефицита AT III, включающие нарушение синтетической функции при хронических заболеваниях печени, недоношенности и потери белков при нефротическом синдроме или асците.
В конце 70-х - середине 80-х гг. ХХ в. появились первые работы, в которых приводились данные результатов исследования АТ III при ДВС-синдроме [13-15]. В этих и последующих публикациях было подтверждено закономерное снижение активности AT III в ходе развития синдрома ДВС вследствие его потребления тромбином и другими плазменными сериновыми протеазами, что создает состояние гепаринорезистентности и способствует прогрессированию тромбинемии [16]. Данный факт имел большое значение для понимания главных механизмов патогенеза ДВС-синдрома и явился основой для разработки принципиально нового направления в интенсивной терапии ДВС-синдрома, основанного на введении больших доз свежезамороженной плазмы и мини- доз гепарина на фоне удаления патологических ингибиторов факторов свертывания крови.

Лечение острого ДВС-синдрома представляет достаточно трудную задачу, так как в организме пациента за весьма короткий период времени развиваются достаточно значимые нарушения в системе гемостаза, которые требуют безотлагательной опережающей интенсивной терапии. В значительной степени исход заболевания зависит от своевременной диагностики ДВС-синдрома и оперативной корригирующей терапии. Последняя, в свою очередь, предусматривает комплексное патогенетическое воздействие на систему гемостаза, гемодинамику, метаболизм и ПОН. Опыт терапии острого ДВС-синдрома, накопленный крупнейшими специалистами в области клинической гемостазиологии, свидетельствует о том, что панацеи в виде какого-либо высокоэффективного препарата не существует. Всегда необходимо обязательное проведение целого комплекса лечебных мероприятий.

Обобщая современные данные литературы, патогенетические принципы интенсивной те- 
рапии ДВС-синдрома можно свести к следующему $[2,3,5,17]$ :

о устранение или уменышение действия провоцирующего пускового фактора - этиотропная терапия (сепсис, шок, травма);

о предупреждение дальнейшего внутрисосудистого свертывания крови;

о элиминация из кровотока микротромбов и агрегатов клеток, активных патологических факторов свертывания - активная детоксикация;

о восстановление нормального функционирования системы гемостаза и реологических свойств крови;

о коррекция нарушений микроциркуляции, водно-электролитных нарушений и кислотнощелочного равновесия;

о устранение проявлений синдрома полиорганной недостаточности $(\mathrm{CПОН})$.

Формирование и прогрессирование ДВСсиндрома во многом зависит от истощения антикоагулянтной системы, а именно системы АТ III, эндогенного гепарина, протеинов С (ПрС) и $\mathrm{S}$ (PS). В связи с этим вполне логичным и правомочным является терапевтический подход, основанный на принципе заместительной антикоагулянтной терапии. Наиболее часто используемым препаратом в данной ситуации является донорская СЗП, при этом объем трансфузии ее должен быть тем больше, чем острее и тяжелее клинические проявления ДВС-синдрома. Эффект СЗП прежде всего связан с присутствием в ней основного антикоагулянта AT III, который является основным физиологическим ингибитором тромбина и противовоспалительным агентом. При этом следует учитывать, что AT III циркулирует в крови в форме, проявляющей низкую ингибиторную активность, в связи с чем для увеличения его активности необходим кофактор, а именно гепарин. Основными механизмами действия комплекса «AT III - гепарин» является блокирование про- цесса трансформации протромбина в тромбин, ингибирование образованного тромбина, а также активированного фактора X (Xa) $[4,10,13]$.

Известно, что на долю АТ III приходится до 80\% всей антикоагулянтной активности плазмы [18-21]. Концентрация АТ III в плазме здорового человека составляет 0,15-0,18 мг/мл, а его активность колеблется в пределах 80-120\% [12]. Период полужизни молекулы АТ III в циркулирующей крови в физиологических условиях составляет около 55-60 ч, но при остром ДВС-синдроме он резко сокращается до 3-4 ч, что всегда следует учитывать при заместительной интенсивной терапии данного состояния. Основным местом синтеза молекулы АТ III является печень, при этом в небольшом количестве он синтезируется в эндотелии и в ткани легкого. Следует отметить, что АТ III является одним из наиболее исследованных ингибиторов плазмы крови. Кроме того, достаточно долгое время его считали основным и единственным антикоагулянтом. Антикоагулянтный әффект AT III прежде всего связан со способностью данного белка ингибировать различные соединения и факторы (рис. 1), участвующие в процессе свертывания крови, и в первую очередь әто тромбин, Ха-фактор, а также XIIa-, Xia-, IХа-факторы.

Необходимо учитывать, что при остром ДВС-синдроме период полужизни АТ III сокращается до 4 ч и менее [12], в связи с чем может возникнуть потребность в перманентном введении концентрата антитромбина под контролем его активности в плазме крови. По некоторым данным, при адекватной коррекции дефицита AT III интенсивность и длительность течения ДВС может быть значительно сокращена, что позволяет добиться существенного повышения выживаемости больных. На ранних этапах развития ДВС, когда снижение активности AT III еще не достигло критического уровня, терапия гепарином может быть еще достаточно 
эффективной. Однако в связи с тем, что антикоагулянтное действие гепарина определяется уровнем активности AT III, при ее снижении до 75\% для достижения сопоставимого эффекта на свертывание гепарина требуется в два, а при активности AT III 50\% в пять раз болыше, чем при нормальном уровне АТ III. С другой стороны, хорошо известно, что сама по себе интенсивная гепаринизация может приводить к дальнейшему снижению уровня активности АТ III, обусловленного увеличением его потребления и активацией липопротеидлипазы [22].

На основании мнений различных авторов можно сделать два основных вывода. Во-первых, поддержание достаточно высокого уровня AT III (зачастую выше нормы) является абсо-

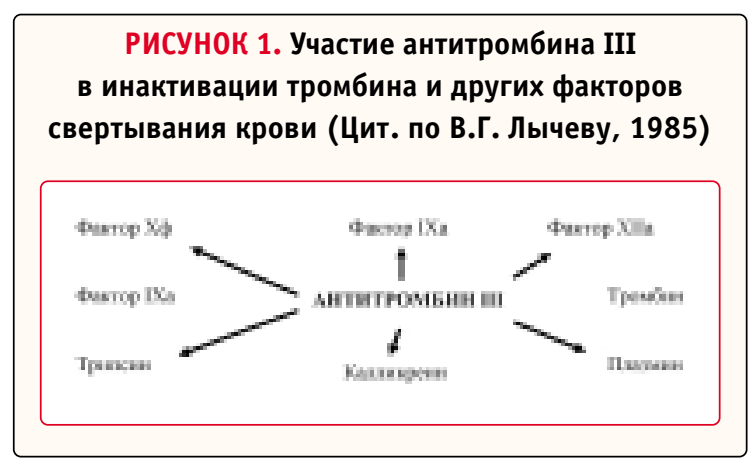

лютно необходимым условием успешной терапии ДВС-синдрома любой әтиологии. Во-вторых, эффективность использования АТ III объясняется, кроме его основного механизма, и его противовоспалительным действием, реализуемого за счет синтеза простациклинов, которые, в свою очередь, уменышают образование свободных радикалов из активированных моноцитов, что уменышает гиперагрегацию тромбоцитов.

В связи с потенциальными проблемами, связанными с применением СЗП, в настоящее время заслуженное внимание привлекает использование концентратов антитромбина III в коррекции дефицита естественных антикоагулянтов. Применение концентрата АТ III в условиях дефицита активности AT III существенно возросло благодаря его способности эффективно восстанавливать циркулирующие количества данного антикоагулянта до нормального физиологического уровня. Поскольку АТ III является основным ингибитором тромбина, представляется особо интересным и патогенетически оправданным использование его препаратов при интенсивной терапии ДВС-синдрома.

В настоящее время фармацевтические компании наладили выпуск лечебных препаратов AT III как из донорской плазмы, так и рекомбинантных [1, 2]. Концентрат AT III был получен из человеческой плазмы и впервые использован в терапевтической практике еще в 1974 г. Препарат концентрата АT III представляет собой стабильный свободный от консервантов лиофилизированный препарат, получаемый из пулированной человеческой плазмы путем сложной переработки с использованием усовершенствованного метода холодового этанолового фракционирования по Кону. Данный препарат пастеризуется нагреванием раствора до 60 љС в течение как минимум 10 ч, что минимизирует риск передачи инфекционных патогенов. Препарат вводится внутривенно в течение 10-20 мин, при этом у пациентов с дефицитом АТ III его уровень возрастает на 1,6-2,1\% на единицу введенного препарата.

Рандомизированное исследование сравнительной эффективности лечения ДВС-синдрома гепарином, концентратом AT III или сочетанием AT III-гепарин показало, что у пациентов с ДВС-синдромом, имевших уровень АТ III 52,6 $\pm 9,7 \%$ от нормы, введение концентратов АT III до восстановления нормального уровня ингибитора было значительно более эффективным, чем лечение только одним гепарином $[14,15]$. 
Однако следует отметить, что у пациентов с массивными травмами сочетание концентрата AT III с гепарином не только не дало дополнительных преимуществ по сравнению с АТ III, но и потребовало достоверно большего объема переливания крови, что объясняется повышенной кровоточивостью при комбинированной терапии [23].

Необходимо учитывать, что восстановление активности AT III снижено у пациентов с тромбозом и ДВС, а также у пациентов, которые находятся на длительной заместительной терапии гепаринами. При введении AT III, меченого радиоактивным изотопом, здоровым людям, его период полувыведения составляет от 2,8 до 4,8 суток. Оптимальная доза введенного препарата рассчитывается исходя из того, что желаемый уровень AT III составляет 80-120\%, а рост активности составляет 1,4\% на 1 ЕД препарата на 1 кг массы тела.

При прогрессировании ДВС-синдрома антитромбин III значительно потребляется, в связи с этим закономерно снижается и его активность. Известно, что снижение уровня активности AT III до 50-60\% является предиктором летального прогноза при ДВС-синдроме, а 20\%ный уровень гарантирует 100\%-ную смертность пациентов. В экспериментах на животных с ДВС-синдромом использование АТ III показало его значимую клиническую эффективность. Так, введение концентрата AT III профилактически или в течение 5 ч после инфицирования животных с формированием септицемии корригировало нарушение коагуляционного профиля и достоверно снижало смертность. Опыт использования AT III у пациентов с острым ДВС-синдромом достаточно трудно интерпретировать, поскольку имеющиеся наблюдения неоднородны по своему дизайну. Данные исследования часто имеют недостаточную выборку, плохо рандомизированы по группам, критериям включения и эффективно- сти, исходному уровню активности АТ III из-за неоднородности клиники и причины ДВС. Однако, несмотря на это, болышинство исследований все-таки демонстрирует улучшение коагуляционных показателей и укорочение периода ДВС крови при использовании концентратов AT III.

Первое крупномасштабное проспективное рандомизированное клиническое исследование использования концентрата АT III при ДВС-синдроме и шоке было проведено Blauhut В. в 1985 г., где было продемонстрировано, что концентрат AT III может быть успешно применен в лечении острого ДВС-синдрома в терапевтических дозах даже без дополнительного назначения гепарина. В проспективном рандомизированном клиническом исследовании, проведенном Vinazzer H.A. (1989), впервые было показано снижение смертности у пациентов с ДВС-синдромом при использовании концентрата AT III (смертность в группе контроля составила 30\% против 14\% в группе с использованием AT III $(p=0,04)$. В результате данного исследования был сделан оптимистический вывод, что адекватная терапия концентратом АТ III приводит не только к сокращению проявлений шока и ДВС, но также увеличивает выживаемость данных пациентов. В дальнейшем этим же автором было продолжено изучение влияния введения концентрата AT III на смертность у пациентов с ДВС крови. В рандомизированном контролируемом исследовании на 170 пациентах (85 пациентов - введение концентрата AT III при его исходной активности ниже 80\%, 85 пациентов - введение гепарина по 300 МЕ/час при постоянной его инфузии) было показано снижение смертности в группе AT III (13\%) против контроля $(31 \%, \mathrm{p}<0,005)$ [14, 15, 23].

Метаанализ терапии концентратом AT III у пациентов с септическим шоком и ДВС-синдромом, проведенный Levi М. и соавт. в 1999 г., 
показал статически значимое снижение смертности от септического шока и ДВС-пациентов, получавших концентрат AT III (47\% в группе контроля и 32\% в группе AT III). Однако и при шоке, не осложненного сепсисом, была выявлена высокая клиническая эффективность концентрата антитромбина. Последующими работами Maki М. и соавт. (1987), Albert J. и соавт. (1992) было показано, что показатели свертывающей системы крови, фибринолиза, а также проявления системного воспалительного ответа нормализовались быстрее при использовании в терапии ДВС-синдрома концентрата АТ III [23].

Влиянию концентрата АТ III на проявления органной дисфункции у пациентов с шоком было посвящено рандомизированное плацебоконтролируемое исследование Inthorn D. и соавт. (1997). Концентрат АТ III вводили под лабораторным контролем его активности в плазме до уровня 120\% в течение 14 суток. Было показано, что введение пациентам концентрата АТ III достоверно снижало частоту возникновения ДВС при шоке $(\mathrm{p}<0,05)$, кроме того, препарат оказывал протективное воздействие на функции легких, печени, почек по сравнению с группой плацебо. Сходные результаты, свидетельствующие о целесообразности применения концентрата антитромбина III в подобных ситуациях, были также продемонстрированы Hinshaw LB в 1990 г. О позитивном влиянии терапии с применением концентрата антитромбина III на состояние пациентов с травмой, отягощенной развитием ДВС-синдрома, также указывали в 2004 г. Miniello S. и соавт. [23].

Существуют отдельные данные, что терапия концентратом AT III предотвращает развитие ПОН и улучшает выживаемость у пациентов, находящихся в критических состояниях. Восстановление уровня AT III в экспериментальной модели ДВС-синдрома адекватно блокирует системную активацию коагуляции, снижает выраженность органной дисфункции и улучшает исход. По данным Ilias W и соавт. (2000), высокие дозы концентрата AT III (30000 ME за четверо суток) при тяжелом сепсисе относительно безопасны и хорошо переносятся пациентами, при этом активность АТ III в плазме повышается с низкого базального уровня до $120 \%$ и остается на этом уровне в течение четырех суток терапии. Авторы изучили некоторые фармакокинетические параметры концентрата AT III у пациентов на фоне тяжелого сепсиса. Так, средний объем распределения концентрата AT III составляет 4,5 л, это означает, что препарат почти не выходит из плазмы в ткани. В ответ на терапию у пациентов с тяжелым сепсисом активность АТ III повысилась в среднем на 1,75\% на 1 ME/кг. В экспериментальных исследованиях, проведенных на животных, были получены доказательства того, что AT III в концентрации, значительно превышающей физиологическую, обладает выраженной противовоспалительной активностью в дополнение к своему антикоагулянтному эффекту. Так, Harada N и соавт. (1999) изучили способность AT III активировать высвобождение простациклина из эндотелиальных клеток и снижать ишемическое/реперфузионное повреждение печени крыс. Было показано, что концентрат AT III снижает реперфузионное повреждение и улучшает микроциркуляцию в зоне ишемии и реперфузии, стимулирует синтез простациклина, который ограничивает взаимодействие между эндотелиальными клетками и нейтрофилами, снижает агрегацию тромбоцитов и продукцию провоспалительных цитокинов. Интересно, что данный әффект нивелируется добавлением гепарина. Антагонизм гепарина обусловлен тем, что АТ III стимулирует продукцию простациклина, связываясь с гепарино-подобными молекулами на мембранах эндотелиальных клеток [1, 2, 23]. 
Эффективность концентрата AT III также определяется методом введения препарата и фармакокинетическими особенностями при различных состояниях. Aibiki М и соавт. (2007) изучили фармакокинетические параметры концентрата AT III у пациентов в критических состояниях. Активность AT III в плазме крови после инфузии препарата у критических пациентов была значительно ниже, чем у пациентов с врожденным дефицитом АТ III. Кроме того, авторы представили доказательства более высокой эффективности введения небольших доз препарата (500 Ед) несколько раз в сутки перед однократным введением всей суточной дозы (1500 Ед).

Было проведено множество экспериментальных исследований на животных, посвященных применению AT III при тяжелом сепсисе. Ostrovsky L. и соавт. (1997) изучили эффекты АТ III на модели ишемического/реперфузионного повреждения кишечника. Авторы применяли высокие дозы концентрата AT III. Предварительное болюсное введение 250 Ед/кг AT III вызвало снижение числа и адгезивных свойств лейкоцитов до начального уровня, а также на 50\% понизило сосудистую проницаемость в зоне повреждения. Данное исследование продемонстрировало, что АТ III может использоваться в терапии для ослабления реакции лейкоцитов на воспаление. Данные множества клинических плацебо-контролируемых исследований также свидетельствуют о целесообразности применения AT III при сепсисе и септическом шоке с целью предотвращения ДВС-синдрома и летального исхода. Авторы рандомизированного двойного слепого плацебо-контролируемого исследования пришли к заключению, что концентрат AT III значимо сокращает частоту сепсис-индуцированного ДВС-синдрома при септическом шоке.

Наконец, в 2000 г. завершилось двойное слепое плацебо-контролируемое мультицент- ровое клиническое исследование KyberSept. В данное исследование было рандомизировано 2314 пациентов с тяжелым сепсисом, у которых проводилось сравнение терапии концентратом AT III (в общей дозе $30000 \mathrm{ME}$ за 4 суток) и плацебо. Результаты исследования показали отсутствие различий в 28-суточной летальности между группами (38,9\% и $38,7 \%, \mathrm{p}=0,94)$. Уровень летальности на 56-е и 90-е сутки, а также летальность в отделении интенсивной терапий не отличались между группами. В подгруппе пациентов, которые не получали в качестве сопутствующей терапии гепарин в течение 4-дневной фазы терапии (698), 28-дневная летальность была ниже в группе терапии AT III - 37,8\% по сравнению с плацебо-группой $-43,6 \%(p=0,08)$, но различие не достигло статистической достоверности. Данная тенденция стала достоверной спустя 90 суток после начала исследования (44,9\% в группе АТ III и 52,5\% в группе плацебо, $p=0,03$ ). У пациентов, получающих концентрат АТ III и сопутствующую терапию гепарином, наблюдалось значительное увеличение частоты кровотечения $(23,8 \%$ и $13,5 \%, \mathrm{p}<0,001)$. В результате исследование было прекращено, и авторы пришли к выводу, что терапия высокими дозами концентрата AT III, начатая в течение первых шести часов после начала заболевания, не влияет на 28-суточную летальность $y$ пациентов с тяжелым сепсисом и септическим шоком. Применение высоких доз концентрата АT III в комбинации с гепарином связано с повышенным риском кровотечения. Есть основания предполагать наличие положительного эффекта терапии концентратом AT III без сопутствующего назначения гепарина.

Результаты данного крупного международного исследования разочаровывали, так как множество доклинических и проспективных клинических исследований фазы II свидетельствовали об эффективности концентрата АT III 
при сепсисе. Более низкая, чем ожидалась, частота снижения активности AT III в момент включения в исследование KyberSept, возможно, повлияла на снижение ожидаемого терапевтического эффекта высоких доз концентрата AT III. Кроме того, как уже отмечалось ранее, для активизации местной антикоагулянтной и противовоспалительной активности AT III должен связаться с гликозаминогликанами на поверхности эндотелия или клеток воспаления. Гепарин конкурентно подавляет связывание AT III с аминогликанами и, таким образом, нивелирует влияние AT III. Данные результаты согласуются с результатами KyberSept, где в подгруппе пациентов, не получавших гепарин, был продемонстрирован положительный эффект высоких доз концентрата AT III. Всем пациентам группы терапии вводилась одинаковая доза концентрата AT III (всего 30000 ME), и неясно, позволяло ли это достичь оптимальной активности AT III у каждого пациента с сепсисом. Независимыми экспертами проводился мониторинг безопасности терапии, который оценивал частоту геморрагических событий. Хотя в группе терапии концентратом AT III наблюдалась большая частота геморрагических эпизодов, смертельные случаи, обусловленные развитием тяжелого кровотечения, отсутствовали в обеих группах. Повышение частоты геморрагических эпизодов ранее не было замечено в клинических контролируемых исследованиях II фазы применения высоких доз концентрата AT III [24, 25].

Анализируя результаты исследования KyberSept, следует отметить, что исследования, показавшие отсутствие положительного эффекта концентрата AT III на выживаемость, были посвящены изучению концентрата AT III у пациентов с сепсисом, а не собственно ДВС-синдромом, т. е. неясно у какой доли пациентов можно было поставить диагноз ДВС-синдрома в соответствии с современными критериями. Так, в
2006 г. исследование Kienast J (2006), посвященное использованию высоких доз концентрата AT III у пациентов с тяжелым сепсисом, где из группы пациентов, участвовавших в KyberSept, были выделены пациенты, имеющие и не имеющие ДВС-синдром, продемонстрировало отсутствие какого-либо влияния препарата на летальность у пациентов без ДВС-синдрома, а при наличии ДВС-синдрома концентрат AT III достоверно снижал летальность на 14,6\% $(\mathrm{p}=0,02)$. Отрицательные эффекты концентрата AT III также зависели от наличия или отсутствия ДВС-синдрома. Так, было показано, что частота геморрагических осложнений при использовании концентрата AT III была выше у пациентов без ДВС-синдрома, а при его наличии она не превышала частоту геморрагических осложнений на фоне плацебо-терапии.

Согласно систематическому обзору исследований, посвященных применению концентрата AT III при ДВС-синдроме на фоне сепсиса, авторы сделали вывод о том, что назначение концентрата AT III, вероятно, предотвращает развитие ПОН и улучшает выживаемость. Однако доступные в настоящее время данные не позволяют оценить безопасность препарата в отношении развития геморрагических осложнений и окончательно оценить его эффективность в клинической практике [23].

Следует отметить, что выбранные для достижения уровня AT III, близкого к 100\%, дозы препарата существенно отличаются в различных исследованиях. В одном из исследований пациенты с ДВС были разделены на группу 50 ЕД/кг/сут и 100 ЕД/кг/сут для достижения сверхнормального уровня активности АТ III более 150\% и более 200\% соответственно. Обе группы продемонстрировали преходящее снижение фрагментов протромбина 1+2 и ТАТ, что было связано с наличием в концентрате гепарина, при этом уровень тромбоцитов и показатели гемостаза оставались неизменны. 
Новый систематический Кохрановский обзор по использованию концентрата АТ III был опубликован Allingstrup M, Wetterslev J, Ravn FB, Mњller AM, Afshari A. в 2016 г. [23]. Данный обзор был опубликован в 2008 г. и обновлен в 2015 г. Основной целью метаанализа явилась оценка влияния концентрата АT III на смертность у критических пациентов и развития побочных явлений на фоне данной терапии. Были проанализированы осложнения, специфические и не относящиеся к данной терапии, кровотечения, воздействие на сепсис и прогрессирование ДВС-синдрома, продолжительность пребывания пациентов в отделении интенсивной терапии (ОИТ) и в больнице в целом. Были проанализированы рандомизированные контролируемые испытания (РКИ) независимо от статуса публикации, даты публикации, состояния ослепления, результатов. Важно отметить, что авторы обзора связались с исследователями, чтобы получить недостающие данные. В целом в обзор было включено 30 РКИ с общим количеством 3933 пациентов (Albert 1992; Balk 1995; Baudo 1992; Baudo 1998; Blauhut 1985; Diaz-Cremades 1994; Eisele 1998; Fourrier 1993; Fulia 2003; Gando 2013; Grenander 2001; Haire 1998; Harper 1991; Inthorn 1997; Kobayashi 2003; Langely 1993; Lavrentieva 2008; Maki 2000; Mitchell 2003; Muntean 1989; Neporada 2008A; Nishiyama 2011; Palareti 1995; Schmidt 1998; Schorr 2000; Schuster 1997; Smith-Erichsen 1996; Vorobyeva 2007; Warren 2001; Waydhas 1998), из них 2 пилотных клинических исследования были проведены в России в г. Архангельске (Neporada-Vorobyeva 2008A; Vorobyeva 2007) $[24,25]$. Анализ смертности в РКИ не показал существенного влияния концентрата АT III изза гетерогенности групп пациентов, при этом анализ подгрупп показал увеличение риска кровотечения при травме и улучшение исхода по СПОН у пациентов с сепсисом в группе концентрата AT III [23].
Таким образом, в течение более чем 15 лет концентрат AT III успешно используется в профилактике и терапии у пациентов с приобретенным дефицитом АT III на фоне острого ДВС-синдрома.

На базе ГБУЗ АО Первая городская клиническая больница им. Е.Е. Волосевич г. Архангельска нами было проведено пилотное исследование по изучению эффективности использования концентрата AT III (Baxter) у пациентов с острым ДВС-синдромом на фоне снижения активности AT III ниже 50\% в реальной клинической практике [24, 25].

\section{МАТЕРИАЛЫ И МЕТОДЫ}

Наше ретроспективное исследование включало анализ реанимационных карт всех пациентов, находившихся в ОАРИТ в период с января по июнь 2008 г. Отбор пациентов для дальнейшего анализа осуществлялся по критериям синдрома системного воспалительного ответа (ССВ): отбирались пациенты, у которых хотя бы в течение суток присутствовали 3 и более признака ССВО. В течение 4 суток с момента развития ССВО данные пациенты оценивались по диагностическим критериям ДВС-синдрома Японской ассоциации критической медицины DIC JAAM (табл. 1), прогностической шкале APACHE II и шкале органной дисфункции SOFA, а также фиксировались лабораторные биохимические и общеклинические показатели; газовый состав артериальной крови; показатели системы гемостаза (уровень фибриногена, АT III, активированное частичное тромбопластиновое время (АЧТВ), международное нормализованное отношения (МНО), количество тромбоцитов. Оценивались объемы гемотрансфузии, назначение антикоагулянтов, сроки пребывания в отделении интенсивной терапии и стационаре, а также летальность на 30-е сутки. На фоне ССВО у пациентов в критических состояниях 


\section{ТАБЛИЦА 1. Шкала ДВС Японской ассоциации критической медицины}

\begin{tabular}{|c|c|c|c|}
\hline Баллы & 0 & 1 & 3 \\
\hline Критерии ССВО & 0 & $1-2$ & $\geqslant 3$ \\
\hline Количество тромбоцитов (*109/л) & $\geqslant 120$ & $\begin{array}{c}\geqslant 80 \text { и <120 } \\
\text { или снижение > 30\% } \\
\text { за } 24 \text { часа }\end{array}$ & $\begin{array}{c}<80 \text { или снижение } \\
>50 \% \text { за } 24 \text { часа }\end{array}$ \\
\hline $\mathrm{MHO}$ & $<1,2$ & $\geqslant 1,2$ & \\
\hline ПДФ (мг/л) & $<10$ & $\geqslant 10 n<25$ & $\geqslant 25$ \\
\hline
\end{tabular}

частота ДВС-синдрома достигает 78\%. Дефицит AT III ниже 50\% встречается у 89\% пациентов с диагнозом ДВС-синдрома. Таким образом, дефицит АТ III, сопровождающий ССBО, может служить в качестве диагностического критерия ДВС-синдрома.

Далее в клиническое проспективное рандомизированное контролируемое исследование был включен 21 пациент в возрасте от 16 до 69 лет массой тела от 50 до 100 кг с установленным диагнозом ДВС-синдрома (по критериям ДВС А - клиническая ситуация, В - клинические симптомы, C - лабораторные маркеры), наличием тромбинемии (D-димер выше 0,5 нг/мл, РФМК выше 14 мг/100 мл) и снижением активности антитромбина ниже 70\%. Были определены следующие критерии исключения: отказ от участия в исследовании; количество тромбоцитов менее 60 x 109/л; продолжающееся кровотечение, вызванное другими, кроме ДВС, причинами; применение ингибиторов фибринолиза, протамина и других гемостатических препаратов. Пациенты были случайно (с использованием метода конвертов) распределены в группу терапии концентратом антитромбина III $(\mathrm{n}=12)$ и СЗП $(\mathrm{n}=9)$.

При использовании СЗП ее трансфузия выполнялась в дозе 10-17 мл на кг веса не позднее 10 минут после размораживания. В группе концентрата АT III препарат вводился при по- мощи инфузомата со скоростью 1000 ME/час в дозе, рассчитываемой по следующей формуле: доза концентрата АТ III [ME] $=(100 \%$ - активность AT III [\%] пациента) х масса пациента [кг].

В рамках исследования назначение перечисленных препаратов производилось в течение 3 суток (в день включения пациента в исследование спустя 24, 48 и 72 ч) в том случае, если уровень АТ ІІІ в плазме крови пациента был ниже 70\%. В качестве антикоагулянтной терапии у 8 пациентов из группы концентрата AT III и 5 пациентов из группы СЗП использовался надропарин в дозе 5700 Ед в сутки и у 4 пациентов из каждой группы - гепарин в дозе 15000 Ед в сутки. Исследование системы гемостаза выполнялось на анализаторе STA-Compact с использованием реактивов STA-Diagnostica. Кровь на исследование забиралась в стандартные гемостазиологические вакутейнеры Vacuette (Gneiner bio-one).

Эффективность лечения оценивалась по следующим критериям: 1) динамика клинических признаков; 2) лабораторные биохимические и общеклинические исследования; 3) оценка газового состава артериальной крови; 4) динамика лабораторных маркеров ДВС (AT III, АЧТВ, МНО, ТВ, РФМК, D-димера, количество тромбоцитов, функциональная активность тромбоцитов); 5) шкалы оценки тяжести состояния пациента APACHE II, SOFA; 6) показатель 
летальности на 14-е и 28-е сутки; 7) сроки госпитализации в отделении интенсивной терапии и стационаре. Безопасность применения концентрата AT III и СЗП оценивалась по частоте возникновения неблагоприятных эффектов (трансфузионные конфликты, аллергические реакции, анафилактический шок, факт заражения инфекцией, передающейся через кровь).

Статистический анализ данных проводился при помощи описательной статистики для количественных, нормально распределенных переменных (двухвыборочный t-тест с различными дисперсиями), непараметрически распределенных переменных (тест Уилкоксона), корреляционный и регрессионный анализ выполнялся с применением ANOVA (коэффициента корреляции Пирсона, rp), двухстороннего точного критерия Фишера (F) с использованием программного обеспечения Excel 5.0 for Windows, и SPSS 11.0 for Windows. Статистически значимыми считались результаты при $\mathrm{p} \leqslant 0,05$.

\section{РЕЗУЛЬТАТЫ ИССЛЕДОВАНИЯ И ОБСУЖДЕНИЕ}

В группу концентрата АТ III было включено 12 пациентов ( $\mathrm{n}=12)$, мужчин - 50\%, средний возраст $42 \pm 5$ лет. Средний срок пребывания в отделении интенсивной терапии (ОИТ) составил $7 \pm 1,6$ суток, а общий срок госпитализации 29,4 \pm 5,8 суток. Летальность в данной группе составила 30\% (n=4). Пациенты группы СЗП (n = 9) (мужчин - 77\%, средний возраст 54,0 \pm 5,8) были старше пациентов первой группы, однако различие не достигло статистической достоверности $(p=0,13)$. Летальность в группе СЗП составила 44\% ( $n=4)$, при этом достоверных различий в уровне летальности между группами не было выявлено (p = $0,67)$. Средний срок лечения в ОИТ не отли- чался от такового в группе АТ III и составил $7 \pm$ 1,3 суток. Тем не менее общий срок госпитализации в группе СЗП был достоверно (p=0,04) ниже и составил 14,6 \pm 3,4 суток. Это разница объясняется полученным в исследовании фактическим различием в летальности в ОИТ: в группе AT III умерло 4 пациента из 12, а в группе плазмы - 4 из 9, и, хотя различие оказалось статистически недостоверным, оно могло повлиять на другие параметры.

При оценке общего состояния на момент включения в исследование количество баллов по прогностической шкале APACHE II у пациентов группы СЗП было достоверно больше, чем у пациентов группы AT III: 16,22 \pm 2,33 и 8,2 \pm 2,32 соответственно ( $p=0,03)$. Средним значениям соответствовал прогнозируемый риск летального исхода 24\% и 9\% соответственно. Данное различие вполне объяснимо небольшим объемом выборки пациентов, включенных в исследование, неоднородностью клинической ситуации, а также большим разбросом такого параметра, как возраст пациентов. В то же время прогнозируемая летальность, определенная при помощи шкалы APACHE II у наших пациентов в обеих группах, была значительно меньше действительной. Это еще раз подтверждает факт, что присутствие ДВС-синдрома значительно ухудшает прогноз, а также точку зрения, что для диагностики ДВС недостаточно общеклинической оценки пациентов, а необходимы специфические признаки и маркеры.

Оценка пациентов по шкале органной дисфункции SOFA при поступлении и в момент включения в исследование была выше в группе СЗП, хотя и статистически недостоверна. Спустя сутки с момента включения в исследование и далее в течение еще 2 дней наблюдений эта разница стала значимой (табл. 2).

Доза концентрата АТ III, вводимого в первые сутки исследования, составила $-750 \pm 75$ Ед, (12 1 Ед/кг). Потребность в введении концен- 
ТАБЛИЦА 2. Выраженность органной дисфункции, оцененная по шкале SOFA, у больных с ДВС-синдромом в разных группах пациентов при поступлении и в период наблюдения

\begin{tabular}{|c|c|c|c|c|c|}
\hline Группы & SOFA & SOFA 0 & SOFA 24 & SOFA 48 & SOFA 72 \\
\hline \multicolumn{6}{|c|}{ Концентрат } \\
\hline AT III & $5,40 \pm 2,1$ & $4,3 \pm 1,3$ & $3,67 \pm 1,3$ & $3,11 \pm 1,4$ & $3,43 \pm 1,7$ \\
\hline СЗП & $7,60 \pm 1,29$ & $8,00 \pm 1,44$ & $7,56 \pm 1,08$ & $7,38 \pm 1,44$ & $9,60 \pm 0,93$ \\
\hline $\mathrm{p}$ & 0,47 & 0,08 & 0,03 & 0,05 & 0,01 \\
\hline \multicolumn{6}{|c|}{$\begin{array}{l}\text { SOFA - оценка по шкале SOFA при поступлении, SOFA O - в момент включения пациента в исследование и SOFA } 24,48,72 \\
\text { спустя } 24,48 \text { и } 72 \text { ч после начала терапии. P - значение р, полученное при сравнении между группами количества } \\
\text { баллов по шкале SOFA в определенные моменты времени. }\end{array}$} \\
\hline
\end{tabular}

трата AT III на вторые сутки исследования возникла у 2 пациентов в дозе 500 и 1000 Ед. В группе СЗП потребность в трансфузии донорской плазмы возникала чаще, у большинства пациентов - ежедневно. Средняя доза СЗП в день включения в исследование составила 684 \pm 53 мл, на второй, третий и четвертый день исследования, соответственно, $700 \pm 87$ мл, 680 \pm 170 мл и $662 \pm 96$ мл. Активность АТ III в плазме у пациентов группы СЗП и концентрата АТ III не отличалась в день поступления в ОИТ $(61,00 \pm 14,53 \%$ и $54,25 \pm 3,15 \%$, соответственно, $p=0,69)$, в момент включения в исследование наблюдалась тенденция $(p=0,08)$ к более низкому уровню активности антитромбина в группе СЗП (45,78 \pm 3,61\% по сравнению с $56,17 \pm 4,37 \%)$. Далее после назначения соответствующей терапии эта разница достигла статистической достоверности, причем более высокая активность AT III в группе концентрата AT III сохранялась в течение всего периода наблюдения (табл. 3). Изучение динамики активности AT III внутри групп показало достоверное ее повышение в группе концентрата AT III уже спустя 2 ч после введения концентрата с последующим сохранением достигнутого уровня активности АТ III. Повторное введение концентрата AT III на вторые сутки потребова- лось у 16\% пациентов ( $\mathrm{n}=2)$. В группе СЗП достоверной положительной динамики активности AT III не наблюдалось. Всем пациентам данной группы потребовалось повторное введение СЗП в течение 3 суток исследования.

Интересно, что функциональная активность AT III сильно коррелировала с количеством тромбоцитов ( $\mathrm{r}=0,79, \mathrm{p}=0,006)$, почасовым диурезом $(\mathrm{r}=-0,69, \mathrm{p}=0,019)$. Отмечена тенденция к отрицательной корреляции между активностью АТ III и уровнем креатинина $(\mathrm{r}=$ $-0,6, p=0,085)$, количеством баллов по шкале SOFA ( $\mathrm{r}=-0,48, \mathrm{p}=0,16)$, уровнем мочевины (моль/л) (r = -0,56, p = 0,073) положительной корреляции с уровнем РФМК ( $\mathrm{r}=0,52, \mathrm{p}=$ $0,12)$, уровнем фибриногена ( $\mathrm{r}=0,56, \mathrm{p}=$ 0,058). Данное наблюдение подтверждает связь между функциональной активностью АТ III и функцией органов, а также показателями гемостаза, являющимися диагностическими маркерами ДВС-синдрома.

Между группами не было обнаружено статистически достоверных различий в количестве тромбоцитов при поступлении и в начальный период наблюдения, только спустя 3 суток с момента включения в исследования количество тромбоцитов в группе концентрата AT III было достоверно больше, чем в группе СЗП 


\section{ТАБЛИЦА 3. Динамика активности АТ III в плазме крови пациентов с ДВС-синдромом в группах концентрата АТ III и СЗП}

\begin{tabular}{|c|c|c|c|c|c|c|c|}
\hline Группы пациентов & AT & AT 0 & AT 2 & AT 6 & AT 24 & AT 48 & AT 72 \\
\hline Концентрат АT III & 54,25 & 56,17 & 71,44 & 69,57 & 71,82 & 74,10 & 74,40 \\
\hline$p^{*}$ & 0,73 & & 0,03 & 0,15 & 0,03 & 0,03 & 0,004 \\
\hline СЗП & 61,00 & 45,78 & 53,60 & 47,33 & 49,78 & 53,50 & 54,38 \\
\hline$p^{* *}$ & 0,41 & & 0,26 & 0,79 & 0,49 & 0,19 & 0,26 \\
\hline$p$ & 0,69 & 0,08 & 0,03 & 0,03 & 0,002 & 0,02 & 0,02 \\
\hline
\end{tabular}

AT - активность АT III в плазме крови в \% при поступлении. АT 2 - активность АT III

в плазме крови в \% спустя 2 ч после начала терапии.

p* - значение p, полученное в группе концентрата AT III при сравнении активности АT III в начальный момент времени

(непосредственно перед началом терапии) с данным показателем в другие моменты времени.

p** - значение р, полученное в группе СЗП при сравнении активности АТ III в начальный момент времени

(непосредственно перед началом терапии) с данным показателем в другие моменты времени.

р - значение р, полученное при сравнении между группами активности АТ III в определенные моменты времени.

$\left(268 \pm 57^{*} 109 /\right.$ л и $119 \pm 37^{*} 109 /$ л соответственно, $\mathrm{p}=0,05)$. Показатели функциональной активности тромбоцитов не отличалась между группами.

Среди тестов, характеризующих систему коагуляции, достоверные различия между группами были найдены только для МНО в момент включения в исследования - показатель был достоверно выше в группе СЗП (1,49 по сравнению $1,15, \mathrm{p}=0,02)$. Внутри групп на фоне лечения не было отмечено достоверной динамики MHO, АЧТВ, ТВ. Динамика уровня фибриногена в группе СЗП проявлялась в повышении его концентрации, и на третьи сутки исследования отличие от исходного значения было значимым $(6,22 \pm 0,46$ г/л по сравнению с 4,40 $\pm 0,73$ г/л, $p=0,053)$. В группе концентрата AT III достоверной динамики уровня фибриногена не наблюдалось. При оценке уровня D-димера статистически значимой динамики его концентрации внутри групп и достоверных различий между группами не было обнаружено. Следует отметить, что исходное состояние сис- темы фибринолиза характеризовалось значимым угнетением эуглобинового лизиса в обеих группах. В группе концентрата AT III наблюдалась положительная динамика в виде тенденции к восстановлению фибринолитической активности (в момент включения в исследование -112 с, спустя 2 суток -88 c, p =0,13). Напротив, в группе СЗП обнаружена тенденция к дальнейшему подавлению фибринолитической активности (в момент включения в исследование -114 с, спустя 3 суток -143 с, $p=$ $0,19)$. Так, на 3-е и 4-е сутки исследования разница в активности фибринолиза между группами была статистически достоверной $(\mathrm{p}=$ 0,03) (табл. 4).

Гемокоагуляционные нарушения напрямую коррелировали с нарушениями метаболизма и органной дисфункцией. Так, показатель эуглобулинового лизиса сильно коррелировал с уровнем креатинина $(r=0,75, \mathrm{p}=0,031)$, парциальным давлением кислорода в артериальной крови $(\mathrm{PaO} 2)(\mathrm{r}=-0,76, \mathrm{p}=0,011)$. Кроме того, выявлена тенденция к корреляции между 
ТАБЛИЦА 4. Динамика фибринолитической активности в группах концентрата АТ III и СЗП

\begin{tabular}{|l|c|c|c|c|}
\hline Группы & Фибринолиз 0 & Фибринолиз 24 & Фибринолиз 48 & Фибринолиз 72 \\
\hline Концентрат АТ ІІІ & 112 & 113 & 88 & 91 \\
\hline СЗП & 114 & 120 & 121 & 143 \\
\hline p & 0,88 & 0,63 & 0,03 & 0,03 \\
\hline $\begin{array}{l}\text { фибринолиз 0- активность фибринолиза в секундах в начальный момент времени. } \\
\text { P-значение р, полученное при сравнении между группами активности фибринолиза в определенные моменты времени. }\end{array}$ \\
\hline
\end{tabular}

фибринолитической активностью и кислородным индексом $(\mathrm{PaO} 2 / \mathrm{FiO} 2)(\mathrm{r}=-0,45 \mathrm{p}=0,19)$, уровнем АСТ Ед/л $(\mathrm{r}=-0,68, \mathrm{p}=0,14)$ и АЛТ Ед/л $(=-0,71, \mathrm{p}=0,11)$ и диурезом (кг/мл/ч) $(\mathrm{r}=-0,6, \mathrm{p}=0,068)$. Этим объясняется сходная динамика показателей функционального состояния органов. Так, при исследовании показателя дыхательной функции - кислородного индекса была выявлена его отрицательная динамика в группе СЗП, что проявлялось в снижении кислородного индекса. И хотя данная динамика внутри группы не была статистически достоверна, разница в кислородном индексе между группами к 3-м суткам исследования достигла статистической значимости ( 0,05) (табл. 5).

Исходные проявления синдрома системного воспалительного ответа (ССВО) не отличались в группах пациентов с ДВС-синдромом. Тем не менее в группе концентрата AT III количество баллов по шкале ССВО снижалось, и на третийчетвертый день исследования разница между группами в данном показателе была статистически достоверной. Оценка пациентов по шкале ДВС ЈААМ перед началом лечения в начальный момент исследования показала статистическую эквивалентность групп (5,9 и 5,6, p = 0,77), а спустя 3 суток оценка по шкале ДВС JAAM в группе пациентов, получавших СЗП, была достоверно выше, чем в группе концентрата AT III (7,3 и 4,1, соответственно, $p=0,02)$. Ни в одной из групп не наблюдались клинически значимые геморрагические осложнения. У 2 пациентов из группы СЗП и 3 больных группы концентрата AT III отмечалась кровоточивость слизистых. Данный клинический симптом может быть связан как с проводимой терапией, так и быть проявлением ДВС.

По нашим данным, снижение активности AT III ниже нормальных значений обладает до-

ТАБЛИЦА 5. Динамика кислородного индекса в группах концентрата АТ III и СЗП

\begin{tabular}{|c|c|c|c|c|c|}
\hline Группы & $\mathrm{PaO}_{2} / \mathrm{FiO}_{2}$ & $\mathrm{PaO}_{2} / \mathrm{FiO}_{2} \mathrm{O}$ & $\mathrm{PaO}_{2} / \mathrm{FiO}_{2} 24$ & $\mathrm{PaO}_{2} / \mathrm{FiO}_{2} 48$ & $\mathrm{PaO}_{2} / \mathrm{FiO}_{2} 72$ \\
\hline AT III & 364 & 359 & 351 & 364 & 341 \\
\hline СЗП & 324 & 306 & 236 & 266 & 229 \\
\hline P & 0,46 & 0,46 & 0,076 & 0,05 & 0,06 \\
\hline \multicolumn{6}{|c|}{$\begin{array}{l}\mathrm{PaO}_{2} / \mathrm{FiO}_{2} \text { - кислородный индекс (мм рт. ст.) при поступлении. } \\
\mathrm{PaO}_{2} / \mathrm{FiO}_{2} \mathrm{O} \text { - кислородный индекс в начальный момент времени. } \mathrm{P} \text { - значение } \mathrm{p}, \text { полученное при сравнении между } \\
\text { группами кислородного индекса в определенные моменты времени. }\end{array}$} \\
\hline
\end{tabular}


ТАБЛИЦА 6. Чувствительность и специфичность дефицита активности антитромбина III в отношении присутствия ДВС-синдрома у пациентов с ССВО (Непорада Е.Л., Воробьева Н.А., 200)

\begin{tabular}{|c|c|c|}
\hline Активность АT III (\%) & Чувствительность (\%) & Специфичность (\%) \\
\hline$\leqslant 80$ & 81 & 26 \\
\hline$\leqslant 50$ & 36 & 85 \\
\hline$\leqslant 35$ & 3 & 96 \\
\hline
\end{tabular}

статочно высокой чувствительностью и низкой специфичностью в отношении присутствия ДВС-синдрома, в то время как выраженное снижение активности AT III ниже 50\% обладает чувствительностью 36\% и специфичностью 85\% (табл. 6).

\section{ВЫВОДЫ}

Терапия концентратом AT III в реальной клинической практике сопровождалась положительной динамикой основных показателей: статистически достоверный рост активности AT III в плазме крови, постепенная активация фибринолитической активности с нормализацией на третьи сутки и тенденция к снижению баллов по шкалам ССВО, ДВС ЈААМ. В группе СЗП не наблюдалось достоверной динамики уровня AT III, фибринолитической активности, и наблюдалась тенденция к увеличению баллов по шкале ДВС ЈААМ и ухудшению показателя газообмена.

В качестве иллюстрации приводим клинический случай использования концентрата антитромбина III при остром ДВС-синдроме на фоне наследственной тромбофилии, осложненной острым мезентериальным тромбозом.

Пациентка Д., 43 г., номер истории болезни 1847, поступила в ОРИТ с диагнозом острый мезентериальный тромбоз. Из анамнеза известно, что пациентка страдает мультифокальным атеротромбозом с поражением коронарных, мозговых и почечных сосудов, в связи с чем месяц назад ей была выполнена операция аортокоронарного шунтирования. Через месяц после хирургической реваскуляризации миокарда, в виду наличия клиники острого тромбоза сосудов кишечника, проведена срочная лапаротомия с резекцией 80 см тонкого кишечника по поводу некроза стенки кишки. В раннем послеоперационном периоде была назначена антикоагулянтная терапия с использованием нефракционированного гепарина 1000 ЕД/ч в виде постоянной инфузии. Через 8 ч введения гепарина было отмечено стойкое снижение активности антитромбина III до 46\%, при этом снижение активности AT III сопровождалось углублением признаков тромбинемии, эндогенной интоксикации. Несмотря на трансфузии донорской СЗП, гепарина, сохранялись выраженные признаки гиперкоагуляции (антитромбин III 53\%, Д-димеры 6,45 нг/л, РФМК 28 мг/100 мл, АЧТВ 28 сек, МНО 0,8) и пареза кишечника ввиду чего в качестве основного антикоагулянта был использован низкомолекулярный гепарин - надропарин в дозе 0,6 мл два раза в сутки. В связи с выраженными признаками перегрузки правых отделов сердца с целью коррекции дефицита антитромбина III была проведена инфузия концентрата антитромбина III в дозе 1000 ЕД.

На фоне данной схемы оптимизации антикоагулянтной терапии удалось стойко стабилизировать показатели системы гемостаза (активность антитромбина III достигла 112\%, Д-димеры снизились до 1,65 нг/л, уровень РФМК снизился до 14 мг/100 мл, АЧТВ 46 сек, МНО 1,2), что клинически сопровождалось улучшением в 
виде появления перистальтики кишечника, уменьшения признаков әндогенной токсемии и разрешения тканевой гипоксии.

На фоне комплексной терапии ПОН, нарушений в системе гемостаза состояние пациентки стабилизировалось, и она была переведена в хирургическое отделение (активность АT III составила 124\%). При генетическом исследова- нии выявлено носительство полиморфизма в гене фибриногена (G/A 455) и тромбоцитов (НРА-1 - А2А2), что свидетельствовало о наследственно-детерминированном нарушении системы гемостаза. Через полтора месяца пациентка была выписана на продленной антитромботической терапии варфарином.

\section{ИСТОЧнИки}

1. Воробьева Н.А. ДВС-синдром - что нового в старой проблеме? Архангельск: Сев. гос. мед. ун-т, 2006. 180 с.

2. Воробьева Н.А. ДВС-синдром в реальной клинической практике. Архангельск: Сев. гос. мед. ун-т, 2015. 232 с.

3. Levi M, Ten Cate H. Disseminated Intravascular Coagulation. N. Engl.J.Med., 1999, 341: 586-592.

4. Баркаган 3.С. Патогенез, диагностика и принципы терапии ДВС-синдрома. Materia Medica, 1997, 1(13): 514.

5. Баркаган 3.С. Узловые вопросы комплексной терапии острого и подострого ДВС-синдрома. Вестн. интенсив. Терапии, 1992, 1: 6-11.

6. Баркаган. 3.С. Геморрагические заболевания и синдромы. М.: Медицина, 1988. 527 с.

7. Abildgaard U. Antithrombin III. Haematologia, 1984, 17(1): 77-79.

8. Asakura H, Takahashi H, Uchiyama T, Eguchi Y, Okamoto K, Kawasugi K, Madoiwa S, Wada H, DIC subcommittee of the Japanese Society on Thrombosis and Hemostasis: proposal for new diagnostic criteria for DIC from the Japanese Society on Thrombosis and Hemostasis. Thrombosis Joumal, 2016, 14: 42.

9. Момот А.П. Патология гемостаза. Принципы и алгоритмы клинико-лабораторной диагностики. СПб.: Формат, 2006, 208 c.

10. Akkawat B, Rojnuckarin P. Protein S deficiency is common in a healthy Thai population. J.Med.Assoc. Thai., 2005, 88(suppl. 4): 249-254.

11. Maclean PS, Tait. RC Hereditary and acquired antithrombin deficiency; epidemiology, pathogenesis and treatment options. Drugs, 2007, 67(10): 14291440.

12. Зубаиров Д.М. Молекулярные основы свертывания крови и тромбообразования. Казань: ФЭН, 2000. 362 с.

13. Takeda K, Yumaushi H. Effects of plasma exchange of acute pancreatitis. 31 St. Congress Intern. Soc. Surgery. Paris, 1985. P. 643.
14. Vinazzer H. Clinical use antithrombin III concentrates. Vox Sand, 1987, 53(4): 193-198.

15. Vinazzer H. Therapeutic use of antithrombin III in shock and disseminated intravascular coagulation. Semin. Thromb. Hemost., 1989, 15: 347-352.

16. Edmunds T, Van Patten S.M., Pollock J. Transgenically produced human antithrombin: structural and functional comparison to human plasma-derived antithrombin. Blood, 1998, 91(12): 4561-4571.

17. Baglin T. Disseminated intravascular coagulation: diagnosis and treatment. BMJ, 1996, 312(7032): 683-687.

18. MacLennan S, Williamson LM. Risks of fresh frozen plasma and platelets.J Trauma, 2006, 60(suppl. 6): 46-50.

19. Баркаган 3.С., Макаров В.А., Лычев В.Г. Новые методы лабораторной диагностики диссеминированного внутрисосудистого свертывания (ДВС-синдрома): Метод. Рекомендации. М., 1989. 23 с.

20. Бышевский А.М., Терсенев О.А. Биохимия для врача. Екатеринбург: Уральский рабочий, 1994. 383 с.

21. Salvesen G, Pizzo SV. Proteinase inhibitors: -macroglobins, serpins, and kunins. Haemostasis and Thrombosis: Basic Principles and Clinical Practice. 3rd ed. Philadelphia, 1994: 241-258.

22. Vorob'eva N, Neporada E, Turundaevskaia O, Mel'nikova G. Place of antithrombin III concentrate in the intensive care of disseminated intravascular coagulation. Anesteziologiia I Reanimatologiia, 2007, 2: 42-4. [PUBMED: 17563999]

23. Allingstrup M, Wetterslev J, Ravn FB, Mњller AM, Afshari A. Antithrombin III for critically ill patients (Review). Cochrane Database of Systematic Reviews, 2016, 2: CD005370. DOI: 10.1002/14651858.CD005370.pub3.

24. Neporada E, Vorobyeva N, Nedashkovsky E. Antithrombin III deficiency correction in patients with disseminated intravascular coagulation. Obshaya Reanimatologia, 2008, 5: 49-54.

25. Neporada E, Turundayevskaya O, Vorobyeva N. Antithrombin III concentrate in comparison with FFP in patients with disseminated intravascular coagulation. Human Ecology, 2008, 7: 47-51. 